\title{
Routing, Modulation Format, Baud Rate and Spectrum Allocation in Optical Metro Rings With Flexible Grid and Few-Mode Transmission
}

\author{
Cristina Rottondi, Pierpaolo Boffi, Paolo Martelli, and Massimo Tornatore
}

\begin{abstract}
Application of few-mode transmission (FMT) in transport optical network is currently under scrutiny, especially for metro networks, where shorter distances and pressing traffic increase (e.g., due to a growing need for metro datacenter interconnection) represent promising conditions for FMT deployment. In this paper, we analyze, from a network-level perspective, the benefits introduced by FMT in metro networks. We consider the application in a flexi-grid network of a FMT system employing hybrid optical/digital mode demultiplexer and low-complexity MIMO-based digital signal processing (DSP). Under this system model, we derive the reach values associated to different modulation formats, baud rates, and number of modes. For the first time to the best of our knowledge, we formulate using linear programming the routing, modulation format, baud rate, and mode assignment problem, for two different switching policies (spatially flexible and spatially and spectrally flexible switching). Using our proposed modes, we identify the configurations that ensure minimum spectrum occupation or minimum cost of installed transceivers, and contrast them to the benchmark case of single-mode transmission.
\end{abstract}

Index Terms-Few-mode optical fibers, RSA problem.

\section{INTRODUCTION}

I S FEW-MODE transmission going to be soon employed in optical transport networks? Surely, the evergrowing Internet traffic requires continuous boost of the transmission capacity and, after extensive research on the gains enabled by advanced modulation formats and by elastic networking in the flexible grid, space has turned out to be the next dimension to increase the capacity of optical transmission systems.

Space Division Multiplexing (SDM) over fiber ribbons, few-mode and multicore fibers has recently attracted a lot of attention from the scientific community and several proofs of concepts have been demonstrated [1]-[3]. Combinations of few-mode transmission over multicore fibers for dense SDM have also been proposed [4]. Fiber ribbons simply package multiple single-mode fibers in a single cable, thus scaling capacity but also the overall cable diameter. Multicore fibers include

Manuscript received May 28, 2016; revised August 26, 2016, October 2, 2016, and November 8, 2016; accepted November 9, 2016. Date of publication November 9, 2016; date of current version December 12, 2016.

C. Rottondi is with the Dalle Molle Institute for Artificial Intelligence, University of Lugano, Lugano 6900, Switzerland, and with the University of Applied Science and Arts of Southern Switzerland, Manno 6928, Switzerland (e-mail: cristina.rottondi@supsi.ch).

P. Boffi, P. Martelli, and M. Tornatore are with the Dipartimento di Elettronica, Informazione e Bioingegneria, Politecnico di Milano, Milano 20133, Italy (e-mail: pierpaolo.boffi@polimi.it; paolo.martelli@polimi.it; massimo. tornatore@polimi.it).

Color versions of one or more of the figures in this paper are available online. an array of physically separated single-mode cores within the same cladding, which must be properly placed in order to minimize intercore crosstalk. Conversely, few-mode fibers enable the transmission of multiple signals over the same wavelength by exploiting different transmission modes (typically vector true modes or LP pseudo modes). This way, the overall fiber capacity could virtually be scaled up by a multiplying factor equal to the number of deployed modes over each wavelength. In particular, in few-mode fibers, the classical notion of spectrum "channel" must evolve to take the spatial dimension into account: modes are organized in groups and each channel may transmit on one or more groups (note that each mode is part of only one group). It follows that, in an optical network supporting flexible grid and few-mode transmission, the Routing and Spectrum Assignment (RSA) problem increases its complexity as we must optimally decide the configuration in terms of: i) group(s) of modes, ii) number of spectrum slots, iii) transceiver baud rates and iv) modulation formats to be attributed to a lightpath. Moreover, in few-mode transmission (FMT), additional transmission impairments have to be accounted for due to the relevant signal coupling among the modes propagating over a wavelength, which leads to high in-band crosstalk and intersymbolic interference. Though the usage of Multiple-Input Multiple-Output (MIMO) techniques at the receiver side can partially mitigate such impairments [5], [6] (by paying the price of higher cost and computational complexity of transceiver hardware, mostly due to increased Digital Signal Processing (DSP)), transmission reaches are reduced w.r.t. single-mode fibers, due to the increase with the distance of the differential mode group delay (DMGD) and hence of the required MIMO complexity (i.e., number of taps). A proposed strategy for obtaining mid-haul [7] and long-haul [8] SDM transmission with realistic MIMO complexity is to use DMGD-compensated links, obtained by combining fiber segments with DMGD of opposite sign. In [9] bi-directional mode assignment is adopted for crosstalk reduction and low complexity MIMO operation. Anyway, as for distance-adaptive modulation assignment in flexible networks [10], also in few-mode transmission a tradeoff emerges between capacity increase and reach decrease, which may significantly impact on Routing and Spectrum Assignment (RSA).

Note that, as of today, forecasting if and when FMT will be employed in operator networks is difficult, but there is generic consensus that the most promising network domain for its application are metro networks, which are currently particularly strained by traffic growth (e.g., due to metro data-center interconnection) and exhibit shorter link distances [11]. 
The aim of this study is to identify, from a network-level perspective, under which conditions FMT becomes beneficial in terms of spectrum/transceiver cost in metro-ring topologies. Our analysis will consider FMT with up to 5 modes, flexigrid spectrum management, and rigorous calculation of reach limitations as a function of different modulation formats and transceiver baud rates assignment. Though some preliminary studies have recently appeared for multicore fibers [12], [13], to the best of our knowledge two of our previous works [14], [15] attempted for the first time to approach the RSA problem in metro networks and provided some initial results on FMT for backbone and metro networks. In those papers, we adopted a hybrid optical/digital mode demultiplexer to separate mode groups in order to minimize the complexity of MIMO-based DSP at the receiver side, limiting cost and consumption. Moreover, we provided detailed reach computations for different modulation formats, considering variable number of modes (up to 5) and impairments due to node traversal.

This paper builds upon our preliminary works and extends them by providing the following novel contributions:

1) we solve the RSA problem considering a new and more scalable channel-based integer linear program.

2) our formulations are extended to capture two SDM spectrum assignments policies: the spatially flexible (SF) and the spatially and spectrally flexible (SSF) scenario.

3) we assess the performance of FMT by analyzing the network configurations that ensure minimum spectrum occupation or minimum cost of installed transceivers, for both the SF and SSF cases, considering single-mode transmission (SMT) as benchmark.

The remainder of the paper is organized as follows: Section II overviews related scientific literature, whereas in Section III we describe the network and transceiver model with reduced MIMO complexity and we discuss how to compute the FMT reaches for different modulation formats. The mathematical formulation used to optimally solve the RSA problem with FMT is presented in Section IV. Numerical assessments are discussed in Section V. Section VI concludes the paper.

\section{SDM OPTICAL NETWORKS}

Several studies on transmission over fiber bundles, few mode and multicore optical fibers have recently appeared (see [2] for a comprehensive survey on SDM techniques).

At network level, some recent studies have addressed planning/resource optimization and control plane solutions for spatially and spectrally flexible optical networks: Refs. [16]-[18] propose different switching approaches for SDM networks, i.e. independent spatial/spectral switching (in which demands can be independently switched in both space and spectrum without restrictions), joint spatial switching (in which only spectral flexibility is allowed and the spatial dimension is switched as a whole), and grouped spatial switching (also called fractionaljoint spatial switching in [19], [20]), in which subgroups of spatial resources can be switched independently. The effectiveness of such approaches is compared in [20]-[22] for networks deploying fiber bundles. In our scenario, we apply the second and third approaches to the case of FMT in our SF and SSF settings. Such approaches correspond to wavelength granularity and fractional space-full wavelength granularity, respectively, according to the classification of WDM-SDM switching schemes in [18, Table 1].

However, only few studies have specifically addressed network-design issues in the context of few-mode/multicore transmission. Among those, Ref. [13] propose an Integer Linear Program (ILP) and a heuristic algorithm for Routing, Spectrum and Core Allocation (RSCA) in flexi-grid networks with multicore fibers, considering intercore crosstalk and minimizing the maximum allocated slot number over the whole network. However, the authors assume the usage of a single modulation format and their proposed model does not support traffic grooming, so that the number of slots to be assigned to each traffic demand is known in advance. Apart from the different fiber technology, note that in our paper we consider multiple modulation formats/baud rates. A variation of the model is proposed in [23] to minimize the overall network cost due to switching modules required for different cores of the input/output links of optical crossconnects.

Ref. [24] proposes an ILP for the CapEx minimized planning of a flexi-grid network with few-mode fibers. The model uses the following assumptions: i) all the modes and wavelengths over a single fiber must be switched as a whole; ii) all the modes over a single wavelength must be switched together. In our model, we apply the latter assumption in the SF scenario, and additionally we consider the SSF case.

Refs. [12], [25], [26] discuss several heuristic algorithms for spectrum and core allocation in a dynamic on-demand scenario for joint reduction of intercore crosstalk and spectrum fragmentation, also in the framework of the Architecture on Demand concept [27]. The proposed methods are based on core/spectrum prioritization and classification criteria, which privilege the assignment of traffic requests to non-adjacent cores or to nonoverlapping spectrum portions in adjacent cores. Refs. [28], [29] propose integer linear models to investigate the impact of MIMO-based crosstalk suppression on the Routing, Wavelength and Core Assignment problem. Refs. [30], [31] apply dynamic routing, spectrum, spatial mode, and modulation format assignment algorithms to evaluate the blocking probability in a few-mode network. In this study we focus on a static network planning scenario.

\section{REACH, TRASMISSION AND NETWORK MODEL}

\section{A. Network Architecture}

We consider as metro-network physical topology a bidirectional ring with a radius of $R \mathrm{~km}, N$ nodes, and $2 N$ optical links. Two links deploying few-mode fibers connect each pair of contiguous nodes (one in clockwise and another one in counterclockwise direction).

Our FMT model is based on the propagation of five optical modes (the fundamental LP01 and the two pairs of spatially degenerate modes LP11a/LP11b and LP21a/LP21b, each one with polarization multiplexing (PM)). We assume the optical spectrum to be partitioned in a grid of frequency slots of width 


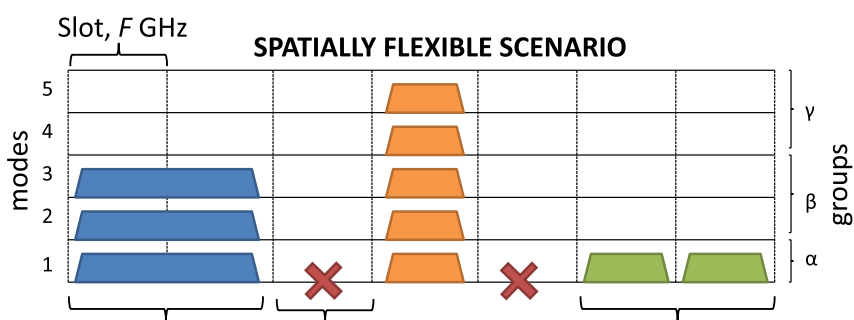

Transceiver optical Guardband, $\mathrm{G}=\mathrm{m} F \mathrm{GHz}$ Superchannel, $\mathrm{b} B \mathrm{GHz}$ bandwidth, $\mathrm{B}=\mathrm{n} F \mathrm{GHz}$

SPATIALLY AND SPECTRALLY FLEXIBLE SCENARIO

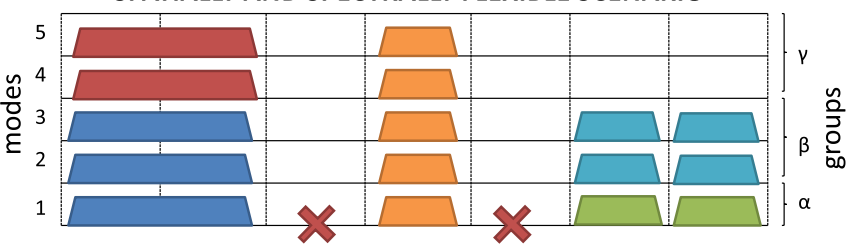

Fig. 1. The considered SDM scenarios.

$F$ GHz. The nominal central frequency $M$ is placed in the middle of the slot, which imposes that optical carriers used to transmit the optical signals are placed at predefined frequencies, regularly spaced along the spectrum every $M=F / 2 \mathrm{GHz}$, as mandated in [32]. The total spectrum width available on each optical link is $S=k F \mathrm{GHz}$, where $k$ is an integer.

If the volume of traffic requests exceeds the maximum capacity that a transceiver can support, traffic demands can be served by transmitting the signals using multiple transceivers. In this case, a channel obtained by contiguously placing multiple transceivers is called "superchannel" and handled by opticalswitching equipment as a single entity [33], provided that each (super)channel is separated from the adjacent ones by a guardband $G=m F \mathrm{GHz}$ (in Fig. 1, we assume $m=1$ ). Such frequency band between neighboring channels prevents overlapping and crosstalk among modulated signals. Note that the superchannel bandwidth can be computed as $b B$, where $b$ is the number of transceivers used to serve the traffic request and $B=n F$ is the transceiver optical bandwidth, expressed as integer multiple of the slot width. In the following, we will refer to $B$ with the term transceiver slot.

Refs. [17], [34] identify two strategies for spectrum assignment in few-mode networks with different degrees of flexibility: the spatially flexible (SF) scenario (Fig. 1, top) assumes that a lightpath can transmit over any mode group, but it does not allow multiple lightpaths to share the same channel on different groups of modes; the spatially and spectrally flexible (SSF) scenario (Fig. 1, bottom) allows two or more lightpaths to share the same channel as long as they use non-overlapping mode groups and occupy the same spectrum width. To capture both scenarios, we adopt the notion of mode group and assume that the five modes are organized in 3 groups: group $\alpha$ includes the LP01 mode, group $\beta$ the LP11a and LP11b modes, group $\gamma$ the LP21a and LP21b modes. For each scenario, the admissible group sets which a lightpath can use are listed in Table I, as well as the group sets which can be attributed to ligthpaths sharing the same channel (in the SSF scenario, up to 3 different mode groups can coexist over the same channel). Note that FMT can
TABLE I

LIST OF ALLOWED MODE GROUP COMBINATIONS

\begin{tabular}{lc}
\hline \hline Scenario & Allowed group combinations \\
\hline SF & $\alpha ; \alpha \cup \beta ; \alpha \cup \beta \cup \gamma$ \\
SSF & $\alpha ; \beta ; \gamma ; \alpha \cup \beta ; \beta \cup \gamma ; \alpha \cup \gamma ; \alpha \cup \beta \cup \gamma$ \\
\hline \hline
\end{tabular}

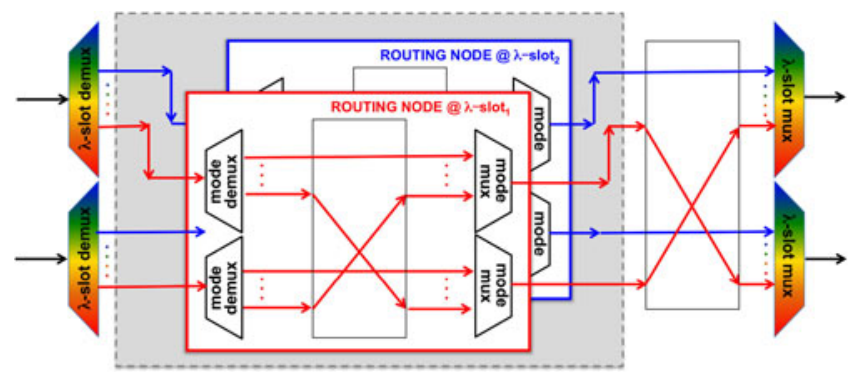

Fig. 2. Node architecture enabling the SSF and SF scenarios, respectively with and without the mode routing represented in the grey inset.

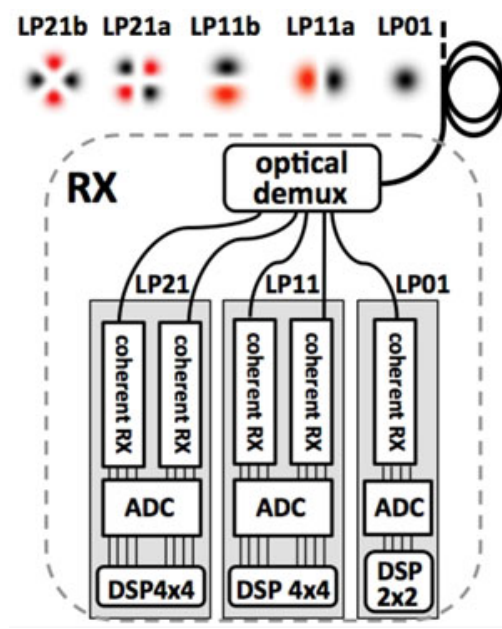

Fig. 3. The considered receiver model.

be downgraded to SMT by considering only group $\alpha$. These two scenarios (SF and SSF) are enabled by the node architecture shown in Fig. 2. In particular, the SF scenario is achieved by dropping out the gray inset, which enables independent mode switching.

\section{B. Transceiver Model}

In FMT, the complexity of MIMO-based DSP must be minimized to avoid expensive and energy-consuming computation. Hence, we adopt the hybrid optical/digital mode separation described in [35], [36] where, using an optical demultiplexer which separates the mode groups, at maximum $4 \times 4 \mathrm{MIMO}$ is employed per mode group, for discriminating the strongly-coupled nearly-degenerate modes of the same group. Our transceiver model is based on the receiver architecture shown in Fig. 3, where the intermodal coupling induced by the propagation sets the more significant limitation to the transmission reach achievable for a given optical signal-to-noise ratio (OSNR) budget. In the few-mode transceiver, an optical de/multiplexer is employed 
TABLE II

MODULATION FORMATS EACHES (KM) FOR 28 AND 14 GBD TRANSCEIVERS AND DIFFERENT AMOUNTS OF TRAVERSED NODES $(j)$

\begin{tabular}{|c|c|c|c|c|c|c|c|c|c|c|c|c|}
\hline \multirow{3}{*}{ Modulation } & \multirow{3}{*}{ Modes } & \multirow{3}{*}{ Groups } & \multicolumn{5}{|c|}{$B_{1}=28 \mathrm{Gbd}, \mathrm{F}=37.5 \mathrm{GHz}$} & \multicolumn{5}{|c|}{$B_{2}=14 \mathrm{Gbd}, \mathrm{F}=25 \mathrm{GHz}$} \\
\hline & & & \multirow[t]{2}{*}{ Bit-rate $(\mathrm{Gb} / \mathrm{s})$} & \multicolumn{4}{|c|}{ Reach $(\mathrm{km})$} & \multirow[t]{2}{*}{ Bit-rate $(\mathrm{Gb} / \mathrm{s})$} & \multicolumn{4}{|c|}{ Reach (km) } \\
\hline & & & & $j=0 *$ & $j=1$ (SSF) & $j=2(\mathrm{SSF})$ & $j=3(\mathrm{SSF})$ & & $j=0^{*}$ & $j=1$ (SSF) & $j=2(\mathrm{SSF})$ & $j=3(\mathrm{SSF})$ \\
\hline \multirow[t]{3}{*}{ QPSK } & 1 & $\alpha$ & 100 & 3200 & 3100 & 3000 & 2900 & 50 & 6900 & 6800 & 6700 & 6600 \\
\hline & $2-3$ & $\alpha \cup \beta$ & $200-300$ & 315 & 215 & 115 & - & $100-150$ & 500 & 400 & 300 & 200 \\
\hline & $4-5$ & $\alpha \cup \beta \cup \gamma$ & $400-500$ & 200 & 100 & - & - & $200-250$ & 280 & 180 & 80 & - \\
\hline \multirow[t]{3}{*}{ 8-QAM } & 1 & $\alpha$ & 150 & 1000 & 900 & 800 & 700 & 75 & 2100 & 2000 & 1900 & 1800 \\
\hline & $2-3$ & $\alpha \cup \beta$ & $300-450$ & 90 & - & - & - & $150-225$ & 150 & 50 & - & - \\
\hline & $4-5$ & $\alpha \cup \beta \cup \gamma$ & $600-750$ & 60 & - & - & - & $300-375$ & 80 & - & - & - \\
\hline \multirow[t]{3}{*}{ 16-QAM } & 1 & $\alpha$ & 200 & 440 & 340 & 240 & 140 & 100 & 1200 & 1100 & 1000 & 900 \\
\hline & $2-3$ & $\alpha \cup \beta$ & $400-600$ & 50 & - & - & - & $200-300$ & 65 & - & - & - \\
\hline & $4-5$ & $\alpha \cup \beta \cup \gamma$ & $800-1000$ & - & - & - & - & $400-500$ & - & - & - & - \\
\hline \multirow[t]{3}{*}{ 32-QAM } & 1 & $\alpha$ & 250 & 205 & 105 & - & - & 125 & 440 & 340 & 240 & 140 \\
\hline & $2-3$ & $\alpha \cup \beta$ & $500-750$ & - & - & - & - & $250-375$ & - & - & - & - \\
\hline & $4-5$ & $\alpha \cup \beta \cup \gamma$ & $1000-1250$ & - & - & - & - & $500-625$ & - & - & - & - \\
\hline \multirow{3}{*}{ 64-QAM } & 1 & $\alpha$ & 300 & 142 & 42 & - & - & 150 & 310 & 210 & 110 & - \\
\hline & $2-3$ & $\alpha \cup \beta$ & $600-900$ & - & - & - & - & $300-450$ & - & - & - & - \\
\hline & $4-5$ & $\alpha \cup \beta \cup \gamma$ & $1200-1500$ & - & - & - & - & $600-750$ & - & - & - & - \\
\hline
\end{tabular}

${ }^{*}$ Columns marked with $j=0$ refer to SMT and FMT either with SF scenario (regardless to the number of traversed nodes) or SSF scenario exclusively for the case of single-link lightpaths. Columns marked with $j>0$ refer only to FMT with SSF scenario.

to separate/combine the mode groups, then the degenerate modes inside each group and their polarizations are digitally demultiplexed after coherent detection. This approach is considered to be a realistic option for few-mode transmission as it allows to significantly increase the capacity, while limiting the increase of hardware cost and DSP complexity, as experimentally demonstrated in [36] by exploiting five spatial modes in addition to two polarizations. Indeed, the FMF used in such experiments supports up to six spatial modes (LP01, LP11a, LP11b, LP21a, LP21b, LP02). However, it is possible to selectively excite only a subset of fiber modes [37], thanks to a proper coupling at the fiber input. In our transmission model we consider five spatial modes (LP01, LP11a, LP11b, LP21a, LP21b) and two polarizations, exactly as in the experiments reported in [36], where only five spatial modes are selectively excited in the FMF and then detected after propagation.

Our model accounts for the intermodal crosstalk accumulated during propagation by deriving a normalized modal crosstalk value (in $\mathrm{dB} / \mathrm{km}$ ) from existing experiments [36], where the realistic coupling between mode groups is measured over 40-km few-mode fiber in presence of a significant number of splices. The intermodal crosstalk induced during the propagation is the main impairment limiting the maximum reach. In fact, we consider only uncompensated links, where the impact of nonlinearities becomes significant only for long-distance transmission [38]. Hence, in our analysis addressed to metro networks, we do not take into account the impact of nonlinearities. Moreover, in reach calculation we have considered only the dominant contribution to the linear intermodal crosstalk, related to the pairs of modes with the strongest coupling. With 5-mode transmission, the modal crosstalk (in $\mathrm{dB} / \mathrm{km}$ ) accumulated over a $L-\mathrm{km}$-long link is calculated as $X T_{\text {reach }}=-26.5+10 \log (L)$, corresponding to the strongest case of coupling between LP11 and LP21 groups [36]. In the case when only the first 3 modes are used, the modal crosstalk (in $\mathrm{dB} / \mathrm{km}$ ) is $X T_{\text {reach }}=-30+10 \log (L)$ [35], corresponding to the LP01 and LP11 coupling.

Table II reports the maximum transmission reaches ensuring the target OSNR required to achieve $4 \cdot 10^{-3}$ BER threshold, as a function of the modulation format (from DP-QPSK to DP- $n$ QAM, with $n=8,16,32,64)$, baud rate $B$ (14 or $28 \mathrm{Gbd})$ and number of modes. We consider a multispan link with an erbiumdoped fiber amplifier (EDFA) for each span, where the span length is $100 \mathrm{~km}$. In particular, to manage the multimode transmission, we consider few-mode EDFAs [39], that is an optical amplifier exploiting erbium-doped FMFs. In the OSNR budget evaluation we assume the same parameters as in [40], assuming the optical noise uniformly distributed over all the spatial modes, with an extra $3 \mathrm{~dB}$ margin to account for the optical mode multiplexer/demultiplexer. In Table II, for each modulation format, the reaches of SMT (i.e. transmission over mode LP01) can be found in the first line (the one marked by group $\alpha$ ) under the column marked by $j=0$. The reaches of FMT in the SF case appear in the second and third lines (marked by $\alpha \cup \beta$ and $\alpha \cup \beta \cup \gamma$, respectively) under the same column $j=0$. In both cases (SMT and FMT-SF), the results do not depend on the number of nodes traversed by the lightpath. Note also that in FMT, when transmitting over modes LP11a-LP11b (group $\beta$ ), for the reach computation we consider the worst-case configuration, i.e. we assume that mode LP01 (group $\alpha$ ) is always occupied. Similarly, when transmitting over modes LP21a-LP21b (group $\gamma$ ), we assume that modes LP01, LP11a and LP11b (i.e. groups $\alpha$ and $\beta$ ) are always occupied. Conversely, when computing the reaches of FMT with SSF scenario, an optical demultiplexer and multiplexer to separate and recombine the mode groups are also required (as shown in Fig. 2). Hence in our transmission model we assume that an EDFA has to be introduced after the node to compensate losses. This further EDFA is not 
considered in case of SF scenario, where only wavelengthselective switching is required (see Fig. 2), because in this case the in-line span EDFA is able to compensate for the total span loss including the wavelength routing loss. Therefore, in the SSF scenario the reach values to be applied depend on the number of nodes traversed by the lightpath: in Table II we report in different columns (marked by $j=0,1, \cdots 3$, where $j$ indicates the number of traversed nodes) the results obtained for lighpaths constituted by up to four links. It follows that the reaches of FMT with SF coincide with the ones of SSF only in the case of single-link lightpaths.

Regarding the evaluation of the reaches, we numerically calculated the OSNR penalty in correspondence to the BER threshold of $4 \cdot 10^{-3}$ by means of Monte Carlo simulations with $2^{17}$ randomly generated symbols, in presence of a random additive white Gaussian noise (AWGN) and a deterministic interferer phase aligned with the signal, which takes into account the impact of intermodal crosstalk, according to [41].

\section{The RMBSA Problem With FMT}

\section{A. Problem Definition and Assumptions}

The classical RSA problem consists in attributing to each traffic request a ligthpath connecting source and destination nodes and in allocating a channel (i.e. a set of contiguous and continuous spectrum slots on each traversed link) for each lightpath. Spectrally-adjacent channels must be separated by a guardband $G$. Additionally, in FMT multiple modes can be activated over a certain spectrum slot. Therefore, constraints to model the spatial flexibility due to spectrum assignment over modes are needed. Finally, transceivers may operate at different baud rates and modulation formats, therefore the best combination of baud rate and modulation format must be chosen for the transceivers of each channel. It follows that the RSA problem evolves into a Routing, Modulation format, Baud rate and Spectrum Assignment (RMBSA) problem.

In this paper, we assume that the ring network is fully transparent, i.e. we do not consider the placement of regenerators nor the presence of space/spectrum converters in the optical nodes. It follows that, in addition to spectral contiguity between slots associated to the same lightpath, spectral continuity constraints must hold for all the links traversed by a lightpath.

\section{B. General RMBSA Problem}

We now present the general RMBSA formulation for an arbitrary network topology and traffic matrix ${ }^{1}$. We adopt a worst case approach to account for transmission impairments due to channel sharing: if two (or more) lightpaths share a channel on at least one link, the reaches of their modulation formats are computed assuming that transmission occurs over the whole set of occupied groups (e.g., if ligthpath 1 transmits over group $\alpha$ and lightpath 2 transmits over group $\beta$, we compute the reaches

\footnotetext{
${ }^{1}$ In Appendix A, we also report a simplified formulation in the case of a single traffic request that will allow us to gain some different, yet relevant insights of the RMBSA problem
}

of their respective modulation formats assuming that they both transmit over 3 modes).

Objective Function

$$
\min \alpha_{1} \sum_{\substack{k \in K, p \in P, m \in M, c \in C, h \in H}} 2 b_{k p}^{c m h} \sigma_{m c h}+\alpha_{2} F \sum_{\left(n, n^{\prime}\right) \in L, s \in S, p \in P} \eta_{n n^{\prime} s p}
$$

Constraints

$$
\begin{aligned}
& \sum_{k \in K} y_{k s d}=1 \quad \forall s \in N, d \in N: t_{s d}>0 \\
& \sum_{\substack{k \in K, n \in N \\
\left(n, n^{\prime}\right) \in L}} y_{k s d} A_{n n^{\prime} k}-\sum_{\substack{k \in K, n \in N \\
\left(n^{\prime}, n\right) \in L}} y_{k s d} A_{n^{\prime} n k} \\
& =\left\{\begin{array}{cl}
1 \text { if } s=i \wedge t^{s d}>0 & \forall n, s, d \in N \times N \times N: s \\
-1 \text { if } d=i \wedge t^{s d}>0 & \neq d \wedge t^{s d}>0
\end{array}\right. \\
& 0 \text { otherwise }
\end{aligned}
$$

$$
\begin{gathered}
y_{k s d} t^{s d} \leq \sum_{\substack{m \in M, h \in H \\
p \in P, c \in C}} b_{k p}^{c m h} \nu_{c} r_{m h} \forall k \in K, s, \\
d \in N \times N: t_{s d}>0
\end{gathered}
$$

$\sum_{m \in M, h \in H} b_{k p}^{c m h} B_{h} \leq\left(W_{p}-G\right) z_{k p c} \quad \forall k \in K, p \in P, c \in C$

$\sum_{m \in M, h \in H} \psi_{k p}^{c m h} \leq z_{k p c} \quad \forall k \in K, p \in P, c \in C$

$\sum_{p \in P, c \in C} z_{k p c} \leq 1 \quad \forall k \in K$

$b_{k p}^{c m h} \leq Q \psi_{k p}^{c m h} \quad \forall k \in K, p \in P, c \in C, m \in M, h \in H$

$\sum_{c \in C} z_{k p c} \leq Q w_{k p} \quad \forall k \in K, p \in P$

$$
\begin{aligned}
& \sum_{k^{\prime} \in K, c \in C: c \neq \alpha} A_{n n^{\prime} k^{\prime}} z_{k^{\prime} p c} \leq Q x_{k p}^{3} \quad \forall k \in K, p \in P,\left(n, n^{\prime}\right) \\
& \in L: A_{n n^{\prime} k}=1
\end{aligned}
$$

$$
\begin{aligned}
& \sum_{\substack{k^{\prime} \in K, c \in C: c=\gamma \\
\vee c=\beta \cup \gamma \vee c=\alpha \cup \beta \cup \gamma}} A_{n n^{\prime} k^{\prime}} z_{k^{\prime} p c} \leq Q x_{k p}^{5} \quad \forall k \in K, p \in P,\left(n, n^{\prime}\right) \\
& \in L: A_{n n^{\prime} k}=1
\end{aligned}
$$

$$
\begin{gathered}
\mathcal{L}_{k} \beta_{k p}^{c m h} \leq \mathcal{L}_{m j h}^{\max }-I_{m j h}^{3} x_{k p}^{3}-I_{m j h}^{5} x_{k p}^{5} \quad \forall k \in K, p \in P, \\
c \in C, m \in M, h \in H, j \in J: j=\sum_{\left(n, n^{\prime}\right) \in L} A_{n n^{\prime} k}
\end{gathered}
$$

$\sum_{k \in K, c \in C} z_{k p c} A_{n n^{\prime} k} \gamma_{c o} \leq 1 \forall p \in P, o \in O,\left(n, n^{\prime}\right) \in L$ 
$\sum_{k \in K: A_{n n^{\prime} k}=1} D_{p s} w_{k p} \leq \eta_{n n^{\prime} s p} \quad \forall s \in S,\left(n, n^{\prime}\right) \in L, p \in P$

$\sum_{p \in P} \eta_{n n^{\prime} s p} \leq 1 \quad \forall s \in S,\left(n, n^{\prime}\right) \in L$

\section{Parameters:}

1) $N:$ set of nodes of the network

2) $L$ : set of physical links $\left(n, n^{\prime}\right)$ of the network

3) $K$ : set of candidate lightpaths (between all node pairs)

4) $H$ : set of transceiver baud rates

5) $C=\{\alpha, \beta, \alpha \cup \beta, \gamma, \beta \cup \gamma, \alpha \cup \beta \cup \gamma\}$ : set of mode group combinations

6) $O=\{\{\alpha, \alpha \cup \beta, \alpha \cup \beta \cup \gamma\}, \quad\{\beta, \alpha \cup \beta, \beta \cup \gamma, \alpha \cup$ $\beta \cup \gamma\}, \quad\{\gamma, \beta \cup \gamma, \alpha \cup \beta \cup \gamma\}\}$ : set of forbidden combinations of mode groups

7) $P$ : set of candidate channels

8) $S$ : set of spectrum slots

9) $M$ : set of possible modulation formats

10) $J=\{0,1,2, \ldots\}$ : set of possible number of intermediate nodes traversed by a lightpath

11) $A_{n n^{\prime} k}$ : boolean parameter, it is 1 if link $\left(n, n^{\prime}\right)$ belongs to lightpath $k$

12) $r_{m h}$ : capacity of one transceiver operating at baud rate $h$ with modulation format $m$

13) $t^{\text {sd }}$ : traffic between source-destination node pairs $(\mathrm{Gb} / \mathrm{s})$

14) $\mathcal{L}_{k}$ : physical length of lightpath $k(\mathrm{~km})$

15) $\mathcal{L}_{m j h}^{\max }:$ maximum reach of modulation format $m$ using baud rate $h$ and traversing $j$ intermediate nodes $(\mathrm{km})$

16) $I_{m j h}^{3}$ : reach reduction (in $\mathrm{km}$ ) for modulation format $m$ and baud rate $h$, when traversing $j$ intermediate nodes using mode group $\beta$ (w.r.t. transmission over group $\alpha$ )

17) $I_{m j h}^{5}$ : reach reduction (in $\mathrm{km}$ ) for modulation format $m$ and baud rate $h$ when traversing $j$ intermediate nodes using mode group $\gamma$ (w.r.t. transmission over group $\alpha \cup \beta$ )

18) $\gamma_{c o}$ : boolean parameter, it is 1 if mode group $c$ belongs to the unfeasible combination $o$

19) $W_{p}$ : spectrum width of channel $p$ expressed as a multiple of $F$ (including guardbands)

20) $G$ : guardband width $(\mathrm{GHz})$, expressed as a multiple of $F$

21) $F_{h}$ : optical bandwidth of transceiver with baud rate $h$, expressed as a multiple of $F$

22) $\nu_{c}$ : number of modes in group $c$

23) $\sigma_{m c h}$ : cost of one transceiver operating at baud rate $h$, modulation format $m$ and transmitting over mode group c. It is computed as $\mathcal{C}\left(1+\left(\nu_{c}-1\right) \delta\right)$, where $\mathcal{C}$ is the cost of a single-mode transceiver and $\delta \leq 1$ is a non-negative tunable multiplicative factor.

24) $D_{p s}$ : boolean parameter, it is 1 if channel $p$ occupies slot $s$

25) $Q$ : positive multiplicative constant, such that $Q>$ $\max \left(|C||K|,|S| / \min _{h \in H} F_{h}\right)$
Variables:

1) $y_{s d k}$ : boolean variable, it is 1 if lightpath $k$ is used to serve the connection request between $(s, d)$

2) $b_{k p}^{c m h}:$ integer non-negative variable indicating the number of transceiver slots dedicated to transceivers operating on mode group $c$ using baud rate $h$ and modulation format $m$ used over lightpath $k$ served by channel $p$

3) $\psi_{k p}^{c m h}$ : boolean variable, it is 1 if transceivers operating on mode group $c$ using baud rate $h$ and modulation format $m$ are deployed over lightpath $k$ served by channel $p$

4) $z_{k p c}$ : boolean variable, it is 1 if lightpath $k$ is established over channel $p$ using mode group $c$.

5) $w_{k p}$ : boolean variable, it is 1 if lightpath $k$ is established over channel $p$

6) $\eta_{n n^{\prime} s p}$ : boolean variable, it is 1 if slot $s$ is used over link $\left(n, n^{\prime}\right)$ within channel $p$

7) $x_{k p}^{3}$ : boolean variable, it is 1 if 3 -mode transmission is used on at least one link of lightpath $k$ over channel $p$

8) $x_{k p}^{5}$ : boolean variable, it is 1 if 5 -mode transmission is used on at least one link of lightpath $k$ over channel $p$

Objective Function: By varying the parameters $\alpha_{1}$ and $\alpha_{2}$, the objective function (1) allows minimizing either $i$ ) the cost of transceivers to be installed (in case $\alpha_{1} \ll \alpha_{2}$ ) or $i$ ) the overall spectrum occupation (in case $\alpha_{1} \gg \alpha_{2}$ ). Note that in case i) if we assign a small positive value to $\alpha_{1}$, the model will still minimize the cost of installed transceivers but in case of multiple solutions, the model will choose the one that occupies the lowest amount of optical spectrum (viceversa for case ii).

Constraints: Constr. (2)-(3) impose that each traffic request between source $s$ and destination $d$ is routed along one of the lightpaths connecting the $(s, d)$ node pair, whereas Constraint (4) imposes that the traffic transmitted along lightpath $k$ accommodated in channel $p$ does not exceed the total capacity of the transceivers allocated within the spectrum portion of the channel. Such capacity depends on the choice of the baud rate $h$, the modulation format $m$ and on the number of modes belonging to the mode group $c$. Moreover, Constr. (5) imposes that the spectrum portion occupied by the transceivers does not exceed the spectral width of channel $p$. Constr. (6)-(7) ensure that a lightpath is deployed on only one channel using only one mode group, whereas Constr. (8) ensures that only one baud rate and modulation format are chosen for trasmission over each deployed ligthpath. Constr. (9) imposes coherence of the values of the variables $z_{k p c}$ and $w_{k p}$ by forcing the lightpath indicator $w_{k p}$ to be 1 if lightpath $k$ is deployed over at least one mode group of channel $p$. Reach limitations are modelled by Constr. (10)-(12): for each lightpath $k$ deployed over channel $p$, Constr. (10) (resp. (11)) activates the boolean variable $x_{k p}^{3}$ (resp. $x_{k p}^{5}$ ) if there exists at least one lightpath $k^{\prime}$ (where $k=k^{\prime}$ is admissible) traversing at least one of the links of $k$ over the same channel $p$ and transmitting over mode group $\beta$ (resp. $\gamma$ ). Such variables are used in Constr. (12), which ensures that, if modulation $m$ and baud rate $h$ are used for transmission over mode group $c$ for lightpath $k$ deployed over channel $p$, then the physical length of $k$ does not exceed the maximum reach of such modulation 


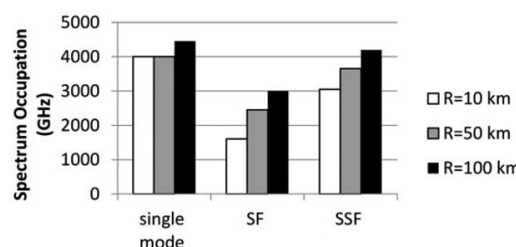

(a)

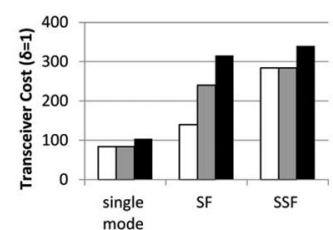

(b)
Fig. 4. Spectrum occupation and transceiver cost obtained when minimizing $S_{o}$ with one-to-all traffic matrix and $10 \mathrm{~Tb} / \mathrm{s}$ overall traffic, assuming $\delta=1$. (a) Minimization of $S_{o}$, (b) $C_{t}$ when minimizing $S_{o}$.

and baud rate combination. Such reach is computed considering the impairments introduced by the traversal of intermediate nodes along the lightpath and by the usage of FMT. As mentioned before, the reach reduction due to FMT is applied based on a worst-case assumption: if the variables $x_{k p}^{3}$ (resp. $x_{k p}^{3}$ and $x_{k p}^{5}$ ) are activated (i.e., FMT is enabled on at least one link of $k$ over channel $p$ ), the reach of each modulation format/baud rate combination is reduced by the amount $I^{3}$ (resp. $I^{3}+I^{5}$ ), which accounts for the impairments due to intermodal crosstalk. Constr. (13) avoids that mode groups sharing at least one mode are adopted for transmission over the same spectrum portion. Constr. (14)-(15) prevent spectrum overlap by imposing that every slot of each link is occupied by at most one channel.

Note that the above presented formulation captures the spatial flexibility allowed by the SSF scenario (discussed in Section III-A), but can be downgraded to the SF scenario by defining the set of mode group combinations as $C=\{\alpha, \alpha \cup$ $\beta, \alpha \cup \beta \cup \gamma\}$ or to SMT by setting $C=\{\alpha\}$.

\section{NUMERICAL ASSESSMENT}

\section{A. Optimization Scenarios}

The RMBSA problem is optimally solved over a ring topology with 8 nodes and radius $R=10,50$ or $100 \mathrm{~km}$. The traffic matrix (with overall traffic volume of 1 or $10 \mathrm{~Tb} / \mathrm{s}$ ) is either all-to-all (i.e. each node sends/receives traffic from every other node) or one-to-all (i.e., traffic is either originated or terminated by one node, which communicates with all the remaining ones). The available spectrum per link is $S=1 \mathrm{THz}$, subdivided in 80 slots of $F=12.5 \mathrm{GHz}$, with $G=F$ (the value of $S$ has been chosen to ensure a good trade-off between computational time and network capacity). Transceivers support DP-QPSK and DP- $n$-QAM, with $n=8,16,32,64$, and can work at two baud rates $(B=14$ or $B=28 \mathrm{GBd}$, occupying $2 F=25 \mathrm{GHz}$ and $3 F=37.5 \mathrm{GHz}$, respectively). In the following, we compare the performance of the SF and SSF scenarios in terms of spectrum occupation $\left(S_{o}\right)$ and overall transceiver $\operatorname{cost}\left(C_{t}\right)$ to a benchmark SMT scenario. Note that the overall spectrum occupation is computed as the total amount of occupied spectral resources over all the links of the network, being thus upper-bounded by $2 N S$.

\section{B. Minimization of Spectrum Occupation}

We start by considering a one-to-all $10 \mathrm{~Tb} / \mathrm{s}$ traffic matrix. Results obtained when minimizing $S_{o}$ are shown in Fig. 4,

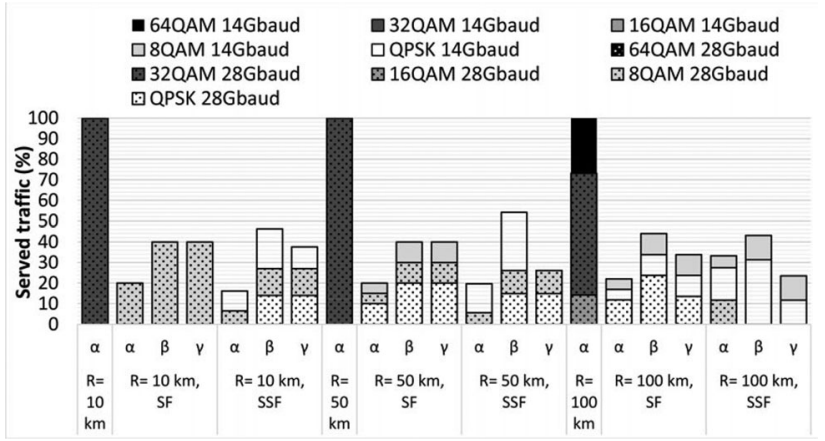

Fig. 5. Percentage of traffic served by each combination of transceiver baudrate, modulation format, and mode group when minimizing $S_{o}$ with one-to-all traffic matrix and $10 \mathrm{~Tb} / \mathrm{s}$ overall traffic, assuming $\delta=1$.

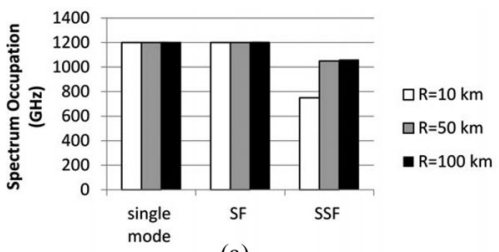

(a)

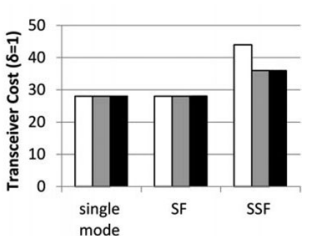

(b)
Fig. 6. Spectrum occupation and transceiver cost obtained when minimizing $S_{o}$ with one-to-all traffic matrix and $1 \mathrm{~Tb} / \mathrm{s}$ overall traffic, assuming $\delta=1$. (a) Minimization of $S_{o}$, (b) $C_{t}$ when minimizing $S_{o}$

whereas the fraction of traffic served by each modulation format over each mode group is plotted in Fig. 5. Fig. 4 a shows that the SF scenario allows for a remarkable reduction of spectrum occupation w.r.t. SMT (32.6\% for $R=100 \mathrm{~km}, 38.8 \%$ for $R=$ $50 \mathrm{~km}$ and $60 \%$ for $R=10 \mathrm{~km}$ ). This is due to a consistent use of mode groups $\beta$ and $\gamma$, which carry around $80 \%$ of the traffic, as detailed in Fig. 5. However, these spectrum savings come at the price of a sharp increase in transceiver cost (that is, e.g., almost 3 times higher than SMT for $R=100 \mathrm{~km}$, see Fig. 4 b). This happens as the modulation formats used in the SF scenario (QPSK and 8-QAM, with QPSK becoming predominant when the ring radius increases) are less advanced than in SMT, due to the shorter reaches of FMT. Note instead that the spectrum savings provided by the SSF scenario are less significant (at most $23.7 \%$ when $R=10 \mathrm{~km}$ ) and become very limited for high ring radius, due to the additional reach penalties associated to independent switch of wavelengths and mode groups (i.e., additional mode demultiplexer affect significantly signal reach). It follows that the SSF scenario suffers a significant increase in the number of transceivers: in fact, even if transceivers are allowed to transmit over different mode groups in the same spectrum portion, the spectrum savings due to the lightpath stacking is not always sufficient to counteract the additional reach penalties of SSF. Furthermore, when comparing the traffic repartition over mode groups in the SF and the SSF scenarios (see Fig. 5), the usage of mode group $\gamma$ in SSF is always less than in SF, showing that 5 mode transmission is heavily impaired by SSF reach reduction.

The advantages of the SSF scenario emerge for lower traffic volumes: results obtained with a one-to-all $1 \mathrm{~Tb} / \mathrm{s}$ traffic matrix (reported in Figs. 6 and 7) show that the $S_{o}$ for SF is equal to SMT, whereas SSF achieves significant savings (up to $37.5 \%$ 


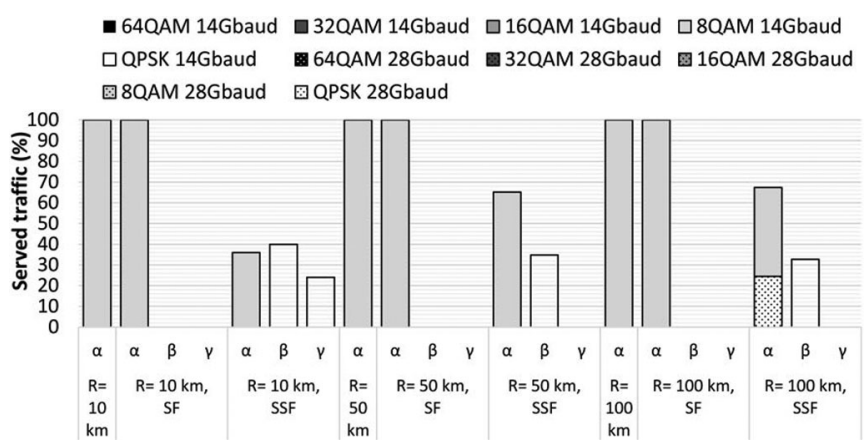

Fig. 7. Percentage of traffic served by each combination of transceiver baudrate, modulation format, and mode group when minimizing $S_{o}$ with one-to-all traffic matrix and $1 \mathrm{~Tb} / \mathrm{s}$ overall traffic, assuming $\delta=1$.

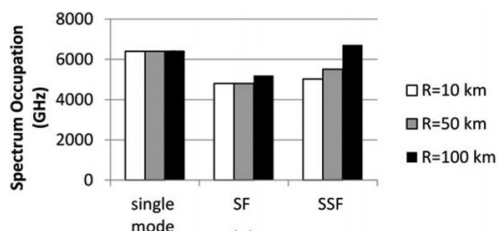

(a)

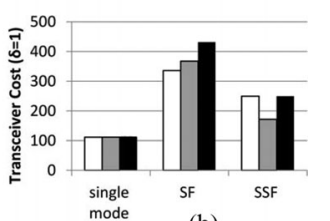

(b)
Fig. 8. Spectrum occupation and transceiver cost obtained when minimizing $S_{o}$ with all-to-all traffic matrix and $10 \mathrm{~Tb} / \mathrm{s}$ overall traffic, assuming $\delta=1$. (a) Minimization of $S_{o}$, (b) $C_{t}$ when minimizing $S_{o}$.

for $R=10 \mathrm{~km})$. In fact, when traffic can be served with few transceiver pairs operating at lower modulation formats (see Fig. 7 which shows that 8-QAM is the preferred format even in SMT), the capability of stacking lighpaths with different source destinations over the same frequency range becomes beneficial. Note that, when minimizing spectrum occupation, $\delta$ has no impact on the minimum $S_{o}$ nor on the distribution of the used modulation formats, but only on $C_{t}$.

Similar results are obtained with an all-to-all $10 \mathrm{~Tb} / \mathrm{s}$ traffic matrix, as reported in Fig. 8. Note that in this case the SSF scenario is even disadvantageous for $R=100 \mathrm{~km}$ and $\delta=1$, where $S_{o}$ exceeds the values obtained in SMT due to the severe reach limitations. However, for $R=10$ and $50 \mathrm{~km}$, the SSF scenario achieves savings comparable to SF, at a much lower transceiver cost. This is motivated by the fact that in this setting SF privileges the adoption of $14 \mathrm{GBd}$ transceivers operating over 3 or 5 modes with 8-QAM or QPSK (which occupy less spectrum resources but increase the number of installed transceivers due to the low spectral efficiency), whereas the SSF opts for $28 \mathrm{GBd}$ transceivers and, when lighpaths are short enough (e.g. they span only one or two links), stacks two lightpaths over the same spectrum portion, using group $\alpha$ for one of the two and group $\beta$ for the other, which results in lower transceiver cost.

Based on these results, few-mode transmission in SF scenario appears a promising alternative to SMT in case of scarcity of spectrum availability due to the consistent occupation savings, whereas in the SSF scenario such savings emerge only for low traffic volumes, i.e., when spectrum scarcity is not an issue, which does not seem to make a good case for SSF adoption in a static-traffic scenario. Nevertheless, further investigations are required to evaluate whether the additional spectral flexibility enabled by SSF could be beneficial in dynamic scenarios, e.g. for reducing spectrum fragmentation.

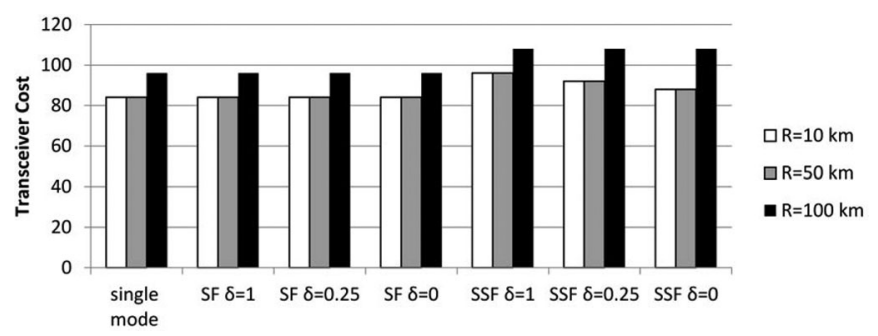

Fig. 9. Minimization of $C_{t}$ with one-to-all traffic matrix and $10 \mathrm{~Tb} / \mathrm{s}$ overall traffic, for various values of $R$ and $\delta$.

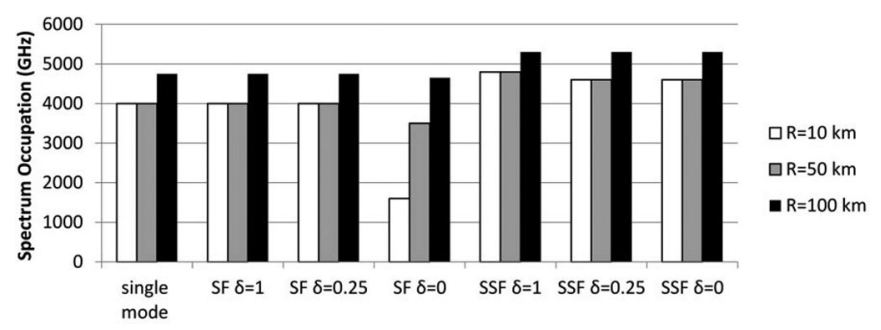

Fig. 10. Spectrum occupation obtained when minimizing $C_{t}$ with one-to-all traffic matrix and $10 \mathrm{~Tb} / \mathrm{s}$ overall traffic, for various values of $R$ and $\delta$.

\section{Minimization of Transceiver Cost}

For transceiver cost $\left(C_{t}\right)$ minimization, when the transceiver cost increases linearly with the number of supported modes (i.e., $\delta=1$ ), the usage of groups $\beta$ and $\gamma$ would be convenient only if such choice reduces the number of transceivers necessary to serve the traffic request w.r.t. SMT, thus compensating the increased cost per transceiver. However, such a case never occurs: considering a given lightpath length, according to the reach values provided in Table II few mode transmission always uses less efficient modulation formats than SMT, thus providing lower spectral efficiency. However, while SMT and SF behave exactly in the same way and show the same performance, the same does not hold for SSF, where in case of multi-hop lightpaths the reaches are further reduced by the additional impairments due to the presence of spatial mux/demux in intermediate nodes. It follows that, even when transmitting over group $\alpha$, less efficient modulation formats have to be used w.r.t. SMT and SF, thus leading to an increase in the number of installed transceivers (and, consequently, in the overall transceiver cost and spectrum occupation).

Conversely, if the cost of a transceiver operating over mode group $\beta$ or $\gamma$ is the same as that of a transceiver operating only over group $\alpha$ (i.e., $\delta=0)^{2}$, few-mode transmission in the case of SF scenario becomes an effective choice to reduce spectrum occupation without increasing the transceiver cost w.r.t. SMT. As reported in Figs. 9 and 10 for the $10 \mathrm{~Tb} / \mathrm{s}$ one-to-all traffic matrix, when $\delta=0$, SF has lower $S_{o}$ than SMT, while maintaining $C_{t}$ unvaried (specifically, $C_{t}=84$ for $R=10$ and $50 \mathrm{~km}$, whilst $C_{t}=96$ for $R=100 \mathrm{~km}$ ). Conversely, the SSF scenario leads to a small increase in $C_{t}$ w.r.t. SMT due to the already discussed reach reductions (specifically, $C_{t}=96$ for

\footnotetext{
${ }^{2} \mathrm{~A}$ sublinear dependence of the cost of the few-mode transceiver on the number of modes used for transmission can be reasonably envisioned, similarly to the case of the multi-wavelength transceiver in WDM optical systems, due to a scalable and compact implementation with cost-optimized package.
} 
$R=10$ and $50 \mathrm{~km}$, whilst $C_{t}=108$ for $R=100 \mathrm{~km}$ when $\delta=1$, whereas $C_{t}=92$ for $R=10$ and $50 \mathrm{~km}$ when $\delta=0.25$ and $C_{t}=88$ for $R=10$ and $50 \mathrm{~km}$ when $\delta=0$ ). For the same reason, even in case of $\delta=0$ the spectrum occupation for SSF is even larger than in SMT.

Based on the above results it follows that, depending on the choice of $\alpha_{1}, \alpha_{2}$ in the objective function (i.e., on the relative importance of spectrum occupation versus transceiver cost minimization) and on the value of $\delta$, the adoption of FMT may be significantly beneficial (when network cost is dominated by spectrum cost) or very undesirable (when network cost is dominated by transceivers) when compared to SMT.

\section{CONCLUSION}

In this paper we studied the behavior of FMT, under both SF and SSF switching assumption, in metro ring networks. To perform this study we first derived FMT optical reaches as a function of the employed modulation format, baud rate and switching approach, and then we formalized the RMBSA as an ILP model. Based on our numerical results over a metro ring topology, we conclude that, when minimizing $S_{o}$, few mode transmission in the SF scenario leads to consistent spectrum savings w.r.t. SMT at the price of a sharp increase in transceiver cost, while in the SSF scenario the benefits of channel sharing are limited to the case of very low traffic. Conversely, when minimizing $C_{t}$, spatial flexibility provides spectrum savings without incrementing the overall transceiver cost w.r.t. SMT only in case the transceiver cost does not increase with the number of modes, (i.e., when the bit-rate/cost ratio for FMT is higher than in SMT), whereas the SSF scenario is always less effective than SMT.

\section{APPENDIX A}

In this appendix we focus on a specific and counterintuitive aspect of FMT: if we take into consideration reach limitations and distance-adaptive modulation formats, for some combinations of offered traffic and transmission distance FMT can be less spectrally efficient than SMT. To analyze this aspect, we solve the RMBSA problem (using a simplified version of our optimization model in Section IV-B, not reported here for sake on conciseness) on a simplified scenario composed of two nodes ( $V_{1}$ and $V_{2}$ ) connected by a single lightpath of length $l$ traversing $j$ intermediate nodes. $T \mathrm{~Gb} / \mathrm{s}$ of traffic are generated by $V_{1}$ and destined to $V_{2}$ and served over a single lightpath. In other words, we want to know what is the combination of modulation format, baud rate and mode group that maximizes spectral efficiency if data must be transferred from $V_{1}$ to $V_{2}$.

The most efficient configuration is then reported in Fig. 11(a) for $j=0$ and Fig. 11(b) for $j=3$ in the SSF case. Note that on the $\mathrm{x}$-axis we vary the values of traffic $T$ from $10 \mathrm{Gbps}$ to $1 \mathrm{Tbps}$ and on the y-axis we vary lightpath lengths up to $4000 \mathrm{~km}$. The color of the various sub-areas in the graph is associated to the modulation format (from white, QPSK, to black, 64-QAM), while the filling pattern indicates the baudrate/mode-group combination.

As we can see in Fig. 11 SMT is always the choice for low volumes (where FMT is simply not necessary to serve the traffic). The advantages of using 3-mode transmission emerge
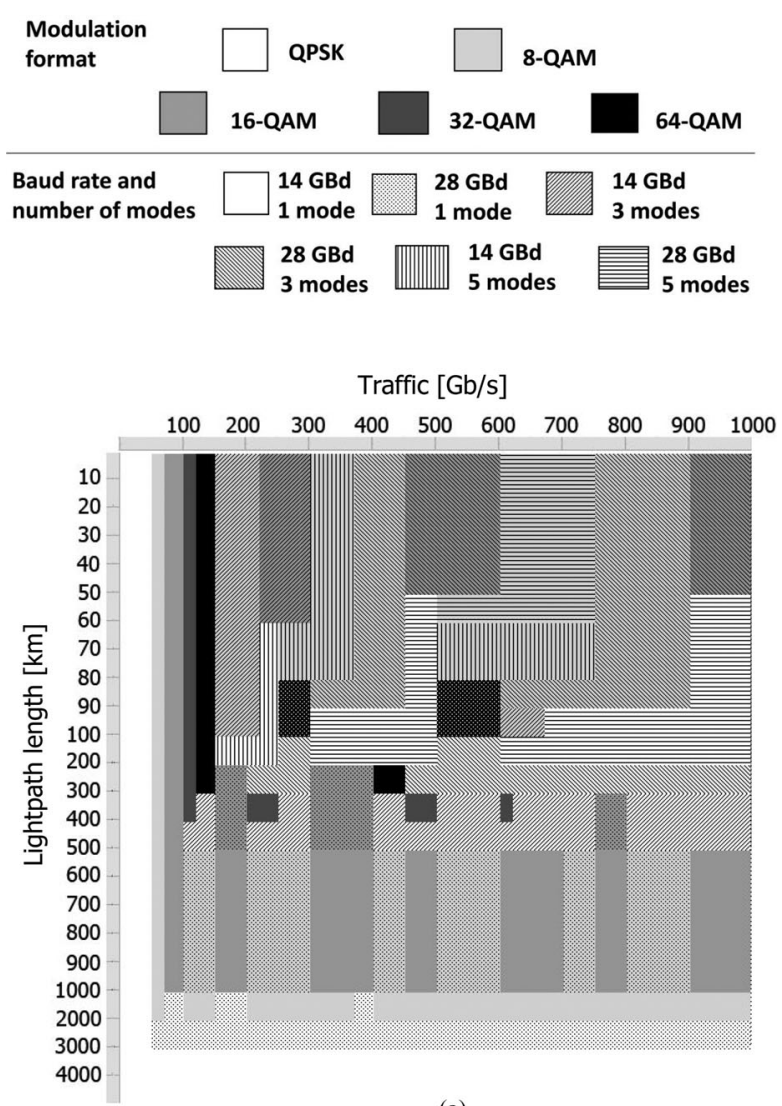

(a)

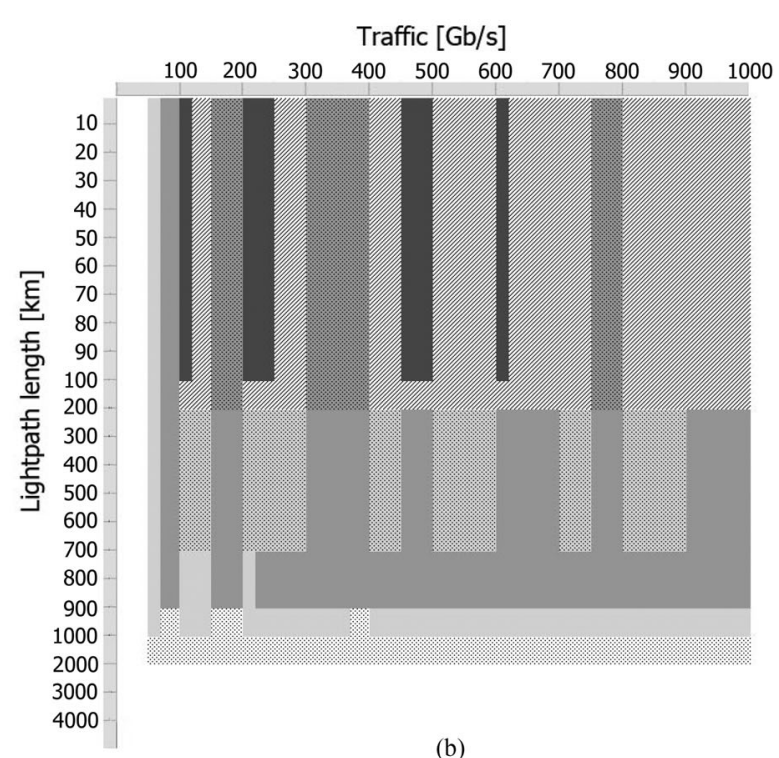

Fig. 11. Optimal baud rate and modulation format assignment in a single lightpath scenario when $\alpha_{1} \gg \alpha_{2} \cdot j=0, j=3$.

for traffic above $150 \mathrm{~Gb} / \mathrm{s}$ and at medium lightpath lengths (up to $500 \mathrm{~km}$ for $j=0$, and up to $200 \mathrm{~km}$ for $j=3$ ), whereas 5-mode transmission is preferred only for very low lengths (up to $200 \mathrm{~km}$ and only for $j=0$ ), in combination with QPSK and 8-QAM modulation formats. However, note that even in case of lighpaths below $200 \mathrm{~km}$, for several values of $T$, 3-modes transmission is more efficient than 5-mode transmission (e.g. for distances below $50 \mathrm{~km}$ ), since it enables the usage of more efficient modulation formats (e.g. 16-QAM). Moreover, when 
the number of traversed nodes increases, for some values of $T$ SMT may still be preferable w.r.t. FMT, since FMT is greatly penalized by the impairments due to node traversal and thus supports only the QPSK format. Conversely, with SMT more efficient modulation formats can be used as 32-QAM with $14 \mathrm{Gbd}$ transceivers (for lightpath lengths up to 100 $\mathrm{km}$ ) and 16-QAM with $28 \mathrm{Gbd}$ transceivers (represented by shaded patterns). Note that $14 \mathrm{Gbd}$ single-mode transceivers are preferred as long as traffic is lower than the capacity of a single transceiver unit. However, such capacity depends on the modulation format in use, which in turn is affected by the lightpath length and the number of intermediate nodes it traverses. Similar considerations can be drawn for the SF case.

\section{REFERENCES}

[1] P. J. Winzer, "Spatial multiplexing in fiber optics: The 10x scaling of metro/core capacities," Bell Labs Tech. J., vol. 19, pp. 22-30, 2014.

[2] D. Richardson, J. Fini, and L. Nelson, "Space-division multiplexing in optical fibres," Nature Photon., vol. 7, no. 5, pp. 354-362, 2013.

[3] G. M. Saridis, D. Alexandropoulos, G. Zervas, and D. Simeonidou, "Survey and evaluation of space division multiplexing: From technologies to optical networks," IEEE Commun. Surveys Tut., vol. 17, no. 4, pp. 2136-2156, Oct.-Dec. 2015.

[4] T. Mizuno, H. Takara, A. Sano, and Y. Miyamoto, "Dense space division multiplexed transmission over multi-core and multi-mode fiber," in Proc. Opt. Fiber Commun. Conf., 2015, Paper Th1D.2.

[5] N. K. Fontaine et al., "30 × 30 MIMO transmission over 15 spatial modes," in Proc. Opt. Fiber Commun. Conf., 2015, Paper Th5C-1.

[6] H. Chen et al., "High spectral efficiency mode-multiplexed transmission over 87-km 10-mode fiber," in Proc. Opt. Fiber Commun. Conf., 2016, Paper Th4C-2.

[7] N. Diamantopoulos et al., "Low DSP complexity mid-haul mode-division multiplexing links utilizing wideband modal dispersion compensated twomode fibers," Opt. Commun., vol. 355, pp. 411-418, 2015.

[8] S. Randel et al., "Mode-multiplexed 6× 20-GBd QPSK transmission over 1200-km DGD-compensated few-mode fiber," in Proc. Opt. Fiber Commun. Conf., 2012, Paper PDP5C-5.

[9] N. P. Diamantopoulos et al., "Mode-unbundled ROADM and bidirectional mode assignment for MDM metro area networks," J. Lightw. Technol., vol. 33, no. 24, pp. 5055-5061, Dec. 2015.

[10] M. Tornatore, C. Rottondi, R. Goscien, K. Walkowiak, G. Rizzelli, and A. Morea, "On the complexity of routing and spectrum assignment in flexible-grid ring networks [invited]," J. Opt. Commun. Netw., vol. 7, no. 2, pp. A256-A267, 2015.

[11] N. K. Fontaine and T. J. Xia, "Will SDM systems ever make sufficient financial sense to justify extensive commercial deployment in terrestrial networks? If so when, where and why?" OFC Workshop, Mar. 2016.

[12] S. Fujii, Y. Hirota, H. Tode, and K. Murakami, "On-demand spectrum and core allocation for reducing crosstalk in multicore fibers in elastic optical networks," J. Opt. Commun. Netw., vol. 6, no. 12, pp. 1059-1071, 2014.

[13] A. Muhammad, G. Zervas, D. Simeonidou, and R. Forchheimer, "Routing, spectrum and core allocation in flexgrid SDM networks with multi-core fibers," in Proc. Int. Conf. Opt. Netw. Des. Modeling, 2014, pp. 192-197.

[14] C. Rottondi, P. Boffi, P. Martelli, M. Tornatore, and A. Pattavina, "Optimal resource allocation in distance-adaptive few-modes backbone networks with flexible grid," in Proc. 2015 Asia Commun. Photon. Conf., Nov. 2015, no. AS4H.2.

[15] C. Rottondi, P. Boffi, P. Martelli, and M. Tornatore, "On the benefits of few-mode transmission in ring metro optical networks with flexible grid," in Proc. Opt. Fiber Commun. Conf., 2016, Paper Tu2H-6.

[16] D. Siracusa, F. Pederzolli, D. Klonidis, V. Lopez, and E. Salvadori, "Resource allocation policies in SDM optical networks," in Proc. Int. Conf. Opt. Netw. Des. Modeling, 2015, pp. 168-173.

[17] D. Klonidis et al., "Spectrally and spatially flexible optical network planning and operations," IEEE Commun. Mag., vol. 53, no. 2, pp. 69-78, Feb. 2015.

[18] D. M. Marom and M. Blau, "Switching solutions for WDM-SDM optical networks," IEEE Commun. Mag., vol. 53, no. 2, pp. 60-68, Feb. 2015.

[19] F. Pederzolli, D. Siracusa, J. M. Rivas-Moscosoy, B. Shariati, E. Salvadori, and T. Ioannis, "Spatial group sharing for SDM optical networks with joint switching," in Proc. Int. Conf. Opt. Netw. Des. Modeling, 2016, pp. 1-6.
[20] P. S. Khodashenas et al., "Comparison of spectral and spatial superchannel allocation schemes for SDM networks," J. Lightw. Technol., vol. 34, no. 11, pp. 2710-2716, Jun. 2016.

[21] B. Shariati et al., "Evaluation of the impact of different SDM switching strategies in a network planning scenario," in Proc. Opt. Fiber Commun. Conf., 2016, Paper Tu2H-4.

[22] D. Siracusa et al., "Spectral vs. spatial super-channel allocation in SDM networks under independent and joint switching paradigms," in Proc. 41 th Eur. Conf. Exhib. Opt. Commun., Valencia, Spain, 2015, pp. 1-3.

[23] A. Muhammad, G. Zervas, G. Saridis, E. Salas, D. Simeonidou, and R. Forchheimer, "Flexible and synthetic SDM networks with multi-corefibers implemented by programmable ROADMs," in Proc. Eur. Conf. Opt. Commun., Sep. 2014, pp. 1-3.

[24] Y. Li, N. Hua, X. Zheng, and G. Li, "Capex advantages of few-mode fiber networks," in Proc. Opt. Fiber Commun. Conf., 2015, Paper Th2A.43. [Online]. Available: http://www.opticsinfobase.org/abstract.cfm?URI=OFC2015-Th2A.43

[25] H. Tode and Y. Hirota, "Routing, spectrum and core assignment for space division multiplexing elastic optical networks," in Proc. 16th Int. Telecommun. Netw. Strategy Planning Symp., Sep. 2014, pp. 1-7.

[26] A. Muhammad, G. Zervas, and R. Forchheimer, "Resource allocation for space-division multiplexing: Optical white box versus optical black box networking," J. Lightw. Technol., vol. 33, no. 23, pp. 4928-4941, Dec. 2015.

[27] S. Fujii, Y. Hirota, T. Watanabe, and H. Tode, "Dynamic spectrum and core allocation with spectrum region reducing costs of building modules in AoD nodes," in Proc. 16th Int. Telecommun. Netw. Strategy Planning Symp., Sep. 2014, pp. 1-6.

[28] Y. Li, N. Hua, and X. Zheng, "Routing, wavelength and core allocation planning for multi-core fiber networks with MIMO-based crosstalk suppression," in Proc. Opto-Electron. Commun. Conf., 2015, pp. $1-3$.

[29] Y. Li, N. Hua, and X. Zheng, "Capex advantages of multi-core fiber networks," Photon. Netw. Commun., vol. 31, no. 2, pp. 228-238, 2016.

[30] R. Proietti et al., "3D elastic optical networking in the temporal, spectral, and spatial domains," IEEE Commun. Mag., vol. 53, no. 2, pp. 79-87, Feb. 2015.

[31] L. Liu, Z. Zhu, and S. Yoo, "3D elastic optical networks in temporal, spectral, and spatial domains with fragmentation-aware RSSMA algorithms," in Proc. Eur. Conf. Opt. Commun., Sep. 2014, pp. 1-3.

[32] International Telecommunication Union, Telecommunication Standardization Sector, "Spectral grids for WDM applications: DWDM frequency grid," ITU-T Rec. G.694.1, Feb. 2012. [Online]. Available: http://www.itu.int

[33] S. Ö. Arık, K.-P. Ho, and J. M. Kahn, "Optical network scaling: Roles of spectral and spatial aggregation," Opt. Express, vol. 22, no. 24, pp. $29868-29887,2014$.

[34] D. Klonidis, P. Zakynthinos, and I. Tomkos, "Opportunities and challenges in the network planning of spatially and spectrally elastic optical networks," in Proc. 17th Int. Conf. Transparent Opt. Netw., 2015, pp. $1-4$.

[35] P. Genevaux, M. Salsi, A. Boutin, F. Verluise, P. Sillard, and G. Charlet, "Comparison of QPSK and 8-QAM in a three spatial modes transmission," IEEE Photon. Technol. Lett., vol. 26, no. 4, pp. 414-417, Feb. 2014.

[36] C. Koebele et al., " $40 \mathrm{~km}$ transmission of five mode division multiplexed data streams at $100 \mathrm{~Gb} / \mathrm{s}$ with low MIMO-DSP complexity," in Proc. Eur. Conf. Expo. Opt. Commun., 2011, Paper Th-13.

[37] P. Boffi, A. Gatto, A. Boletti, P. Martelli, and M. Martinelli, " 12.5 gbit/s VCSEL-based transmission over legacy MMFs by centre-launching technique," Electron. Lett., vol. 48, no. 20, pp. 1289-1290, 2012.

[38] P. Boffi et al., "PDM-16QAM transmission performance over uncompensated fiber links," Opt. Express, vol. 19, no. 22, pp. 21 898-21 903, 2011

[39] G. Le Cocq et al., "Modeling and characterization of a few-mode EDFA supporting four mode groups for mode division multiplexing," Opt. Express, vol. 20, no. 24, pp. 27 051-27 061, 2012.

[40] C. Rottondi, M. Tornatore, and G. Gavioli, "Optical ring metro networks with flexible grid and distance-adaptive optical coherent transceivers," Bell Labs Tech. J., vol. 18, no. 3, pp. 95-110, 2013.

[41] P. Winzer, A. Gnauck, A. Konczykowska, F. Jorge, and J.-Y. Dupuy, "Penalties from in-band crosstalk for advanced optical modulation formats," in Proc. Eur. Conf. Expo. Opt. Commun., 2011, Paper Tu-5. 